\title{
A REMARK ON THE BOX-COUNTING DIMENSION OF THE SINGULAR SET FOR THE NAVIER-STOKES EQUATIONS*
}

\author{
WITOLD SADOWSKI ${ }^{\dagger}$
}

\begin{abstract}
Let $u$ be a suitable weak solution of the Navier-Stokes equations and let $\mathcal{S}$ be the set of its singular points in space-time. We prove that if $u_{t}$ is square integrable then the box-counting dimension of $\mathcal{S}$ is no larger than one.
\end{abstract}

Key words. Navier-Stokes equations, singular set, partial regularity, box-counting dimension.

AMS subject classifications. 35K55, 35Q30, 35Q35.

\section{Introduction}

In this paper we consider the Navier-Stokes equations for an incompressible fluid:

$$
u_{t}-\Delta u+(u \cdot \nabla) u+\nabla p=0, \quad \operatorname{div} u=0,
$$

where $u=\left(u_{1}, u_{2}, u_{3}\right)$ is the velocity of the fluid and $p$ is the pressure. The domain of the flow is an open set $\Omega \subseteq \mathbb{R}^{3}$. There is no external force acting on the fluid. We consider the problem (1.1) with an initial condition $u_{0}$ :

$$
u(\cdot, 0)=u_{0} .
$$

Throughout the paper we assume that $u_{0} \in V$, where $V$ is the completion of smooth, divergence-free functions with compact support in the norm of $H^{1}$.

The only global in time solutions of the system (1.1)-(1.2) that have been constructed to date are weak solutions (Leray [13], Hopf [6]). Unfortunately, such solutions are not known to be unique and regular. As a result the existence of singular times $^{1}$ and of singular points in space-time ${ }^{2}$ has not been ruled out. However, many results show that both the set of singular times and the set of singular points in spacetime are rather "small". The smallness of these sets is usually measured in terms of the Hausdorff and the box-counting dimensions (for definitions of these dimensions see the next section). In particular, one can construct weak solutions to (1.1)-(1.2) that are smooth on an open set $\Omega \times \mathcal{R}$, where $\mathcal{R}=(0, \infty) \backslash \mathcal{T}$ and $\mathcal{T}$ is the "small" set of singular times (see for example Heywood [5]). Both the Hausdorff and the box-counting dimensions of $\mathcal{T}$ can be bounded above by the same number:

$$
d_{H}(\mathcal{T}) \leq 1 / 2 \text { and } d_{B}(\mathcal{T}) \leq 1 / 2
$$

(see Leray [13] and Scheffer [18] for the Hausdorff dimension and Robinson \& Sadowski [14] for the box-counting dimension).

The set $\mathcal{S}$ of singular points in space-time was first investigated by Scheffer [18] and then addressed in a seminal paper of Caffarelli, Kohn, and Nirenberg [1]. The Liu.

*Received: May 10, 2011; accepted (in revised form): July 23, 2012. Communicated by Chun $\dagger \mathrm{F}$ 2, 02-097 Warszawa, Poland (W.Sadowski@mimuw.edu.pl).

This work was supported by EPSRC grant EP/G007470/1 and Polish Government grant N N201 547438 .

${ }^{1} \mathrm{~A}$ time $t$ is called a singular time of $u$ if $\|D u(s)\|:=\|D u(\cdot, s)\|_{L^{2}(\Omega)}$ is unbounded in every neighbourhood of $t$.

${ }^{2} \mathrm{~A}$ point $z \in \mathbb{R}^{4}$ is called a singular point of $u$ if $u$ is unbounded in every neighbourhood of $z$. 
authors considered a special class of weak solutions called 'suitable weak solutions'. Such solutions have two additional properties: they satisfy a local energy inequality and their associated pressure $p$ belongs to $L^{3 / 2}$. For such solutions it can be proved that the Hausdorff dimension of $\mathcal{S}$ is no greater than 1. It can also be deduced from the proof that the box-counting dimension of the singular set is no greater than $5 / 3$ (see for example Robinson \& Sadowski [15]). The gap between the upper bounds for both dimensions:

$$
d_{H}(\mathcal{S}) \leq 1 \text { and } d_{B}(\mathcal{S}) \leq 5 / 3
$$

is the outcome of different criteria for regularity that are used in the proofs of the above bounds. The bound for the Hausdorff dimension of the singular set follows from the fact that there exists a constant $\varepsilon>0$ such that if

$$
\limsup _{r \rightarrow 0} \frac{1}{r} \int_{Q_{r}(x, t)}|\nabla u|^{2} \leq \varepsilon,
$$

then $(x, t)$ is a regular point of $u$. Here $Q_{r}$ is the space-time cylinder (see the next section for notation). On the other hand, the upper bound on the box-counting dimension is a simple corollary from the following criterion for regularity. If for some sufficiently small $\varepsilon>0$ we have

$$
\frac{1}{r^{2}} \int_{Q_{r}(x, t)}|u|^{3}+|p|^{3 / 2} \leq \varepsilon,
$$

then $u$ is regular in a neighbourhood of $(x, t)$.

The gap between the two upper bounds on $d_{B}(\mathcal{S})$ and $d_{H}(\mathcal{S})$ was recently reduced by Kukavica [10], who proved that in fact the box-counting dimension of a singular set in space-time is no greater than $135 / 82$ :

$$
d_{B}(\mathcal{S}) \leq \frac{135}{82} \approx 1.646
$$

No better upper bounds on the box-counting dimension of the singular set $\mathcal{S}$ are known. The problem of closing the gap between upper bounds on $d_{B}(\mathcal{S})$ and $d_{H}(\mathcal{S})$ seems to be even more interesting if we recall that there are sets of Hausdorff dimension zero whose box-counting dimension can be arbitrarily large. For example a set $X_{\alpha} \subset$ $l^{2}$ given by $X_{\alpha}=\{0\} \cup\left\{n^{-\alpha} e_{n}\right\}_{n=1}^{\infty}$ has box-counting dimension $\alpha^{-1}$ and Hausdorff dimension 0 , since it is countable (see Robinson [17] for more details).

In this paper we prove that if the classical time derivative, which due to the epochs of regularity property is defined almost everywhere (namely, on the set $\Omega \times \mathcal{R}$ ), is square integrable in the space-time, then not only the Hausdorff but also the boxcounting dimension of the singular set is no greater than 1 .

\section{Notation}

By $B_{r}(x)$ we denote a ball of radius $r$ centered at $x$. A space-time cylinder is denoted by $Q_{r}(x, t)$ :

$$
Q_{r}(x, t)=\left(t-r^{2}, t\right) \times B_{r}(x) .
$$

The set of singular times $\mathcal{T}$ is the set of all times $t>0$ such that

$$
\limsup _{s \rightarrow t}\|D u(s)\|=\infty .
$$


A point $z=(x, t) \in \mathbb{R}^{3} \times \mathbb{R}$ is called regular if $u$ is bounded in some neighbourhood of $z$. A point $z$ is called singular if it is not regular.

REMARK 2.1. In what follows we will use the fact (see Skalak and Kučera [20]) that for each regular point $z=(x, t)$ one can find a cylinder $(t-\epsilon, t+\epsilon) \times B_{\delta}(x)$ such that for almost all $\tilde{x} \in B_{\delta}(x)$ the function $u(\tilde{x}, \cdot)$ is absolutely continuous on $(t-\epsilon, t+\epsilon)$.

The set of all singular points in space-time is denoted $\mathcal{S}$. We also define the projection of $\mathcal{S}$ onto $\mathbb{R}^{3}$ :

$$
\Pi \mathcal{S}=\left\{x \in \mathbb{R}^{3}:(x, t) \in \mathcal{S} \text { for some } t \in(0 ; \infty)\right\} .
$$

Let $X$ be a compact set in $\mathbb{R} \times \mathbb{R}^{3}$. The parabolic Hausdorff dimension of $X$, denoted by $d_{H}(X)$, is given by

$$
d_{H}(X)=\inf \left\{s \geq 0: \mu^{s}(X)=0\right\},
$$

where $\mu^{s}(X)$ denotes parabolic Hausdorff measure:

$$
\mu^{s}(X)=\lim _{\delta \rightarrow 0} \inf \left\{\sum_{i=1}^{\infty} r_{j}^{s}: X \subset \bigcup_{j=1}^{\infty} Q_{r_{j}}\left(x_{j}, t_{j}\right), r_{j}<\delta\right\} .
$$

The box-counting dimension of $X$, denoted by $d_{B}(X)$, is defined by the formula

$$
d_{B}(X)=\limsup _{\epsilon \rightarrow 0} \frac{\log N(X, \epsilon)}{-\log \epsilon}
$$

where $N(X, \epsilon)$ is the maximum number of disjoint balls of radius $\epsilon$ centered at points of $X .^{3}$

\section{Main result}

Little is known about regularity of the time derivative of a weak solution $u$ of the Navier-Stokes equations. It can be shown that $u_{t} \in L^{4 / 3}\left(0, T ; H^{-1}\right)$ (see for example Galdi [4]), but even the question whether $u_{t}$ is continuous in a neighbourhood of a regular point is an open problem (see Skalak and Kučera [20]). On the other hand, some conditional results about regularity of the Navier-Stokes equations involve assumptions on $u_{t}$. For example, Serrin ([19]) proved that if in addition to his criterion for regularity ${ }^{4}$ we also have $u_{t} \in L^{p}$ with $p>1$, then the space derivatives of $u$ are absolutely continuous functions of time. Kohn in [8] showed how the assumption $u_{t} \in L^{3 / 2}$ leads to a simplified proof of the partial regularity theorem by Caffarelli, Kohn, and Nirenberg [1]. Below we prove a conditional result on the box-counting dimension of the singular set making an assumption on the integrability of $u_{t}$. We use the fact that $u_{t}$ is defined in a classical way for all $t \in \mathcal{R}$.

TheOREM 3.1. Let u be a suitable weak solution of the Navier-Stokes equations (1.1)(1.2) with $u_{0} \in V$ and $\Omega=\mathbb{R}^{3}$. Moreover, let $u_{t}$ be the classical time derivative of $u$ defined on $\mathbb{R}^{3} \times \mathcal{R}$. If $u_{t}$ is square integrable then the box-counting dimension of the singular set is no greater than 1 .

\footnotetext{
${ }^{3}$ In an equivalent (and more popular) definition $N(X, \varepsilon)$ denotes the minimum number of balls of radius $\varepsilon$ needed to cover the set $X$ (see Falconer [2]).

${ }^{4}$ Serrin's criterion states that if $u \in L^{r}\left(t_{1}, t_{2} ; L^{p}\left(B_{\delta}(x)\right)\right)$ with $2 / r+3 / p<1$, then $u$ and its space derivatives are uniformly bounded on compact subsets of the space-time cylinder.
} 
Proof. Let us begin with some remarks on the boundedness of the set $\mathcal{S}$. Since $u_{0} \in V$ a weak solution $u$ is regular on some time interval $\left(0, T^{*}\right)$. Moreover, the set $\mathcal{T}$ (see for example Heywood [5]) is bounded so $u$ is regular for sufficiently large times. Therefore $\mathcal{S} \subset \Omega_{T}$ where

$$
\Omega_{T}:=\left(T_{1}, T_{2}\right) \times \mathbb{R}^{3},
$$

for some $T_{1}, T_{2}>0^{5}$.

We claim that $u \in W^{1,2}\left(\Omega_{T}\right)$. As we already know that $u$ and its space derivatives belong to $L^{2}\left(\Omega_{T}\right)$ we only need to check that $u_{t}$ is well defined as a distributional derivative over the space-time domain.

To this end we notice that $u_{i}(x, \cdot), i=1,2,3$ is absolutely continuous on $\left[T_{1}, T_{2}\right]$ for almost all points $x \in \mathbb{R}^{3}$. Indeed, take any $R>0$ and consider a closed ball $\bar{B}(0, R)$. It is enough to show that for almost all $x \in \bar{B}(0, R)$ the function $u_{i}(x, \cdot), i=1,2,3$ is absolutely continuous on the interval $\left[T_{1}, T_{2}\right]$. Take any sufficiently small $\varepsilon>0$. Since the box-counting dimension of the singular set is less than $5 / 3$ we can cover the set $\Pi \mathcal{S} \cap \bar{B}(0, R) \subset \mathbb{R}^{3}$ with a finite collection $\mathcal{C}$ of open balls of total measure $\varepsilon$. Now choose any point $\tilde{x}$ in the compact set $\bar{B}(0, R) \backslash \mathcal{C}$. The line segment $I_{\tilde{x}}=$ $(\tilde{x}, t), t \in\left[T_{1}, T_{2}\right]$ consists only of regular points. Simple compactness arguments and the remark in the previous section show that we can cover the points of $I_{\tilde{x}}$ with a finite number of cylinders $\left\{Q_{r_{i}}\left(\tilde{x}, t_{i}\right)\right\}, i=1,2, \ldots, N$ such that $u(x, \cdot)$ is absolutely continuous on $\left(t_{i}-r_{i}, t_{i}+r_{i}\right)$ for almost every $x \in Q_{r_{i}}\left(\tilde{x}, t_{i}\right), i=1,2, \ldots N$. Therefore for each point $\tilde{x} \in \bar{B}(0, R) \backslash \mathcal{C}$ we can find an open ball $B_{\delta}(\tilde{x})$ such that for almost all $x \in B_{\delta}(\tilde{x}), u_{i}(x, \cdot)$ is absolutely continuous on $\left[T_{1}, T_{2}\right]$. We choose finite subcovering of $\bar{B}(0, R) \backslash \mathcal{C}$ to conclude the assertion.

As $u$ is absolutely continuous on almost all lines parallel to the axis ( $u$ is ACL) and $u$ with its derivatives belong to $L^{2}\left(\Omega_{T}\right)$, we invoke Lemma 5.6.2 from Kufner et al. [9] to deduce that $u \in W^{1,2}\left(\Omega_{T}\right)$. From the Sobolev embedding theorem it follows then that $u \in L^{4}\left(\Omega_{T}\right)$. From the Calderon-Zygmund inequality we conclude that $p \in L^{2}\left(\Omega_{T}\right)$. Now the result follows from Theorem 1 in Robinson and Sadowski [16], where a more general case was treated. However, in the case considered here the argument may be substantially simplified, so we present this simpler version below.

Choose sufficiently small $r>0$ and let $\mathcal{C}_{r}$ be a set consisting of the maximal number of 4-dimensional balls of radius $2 r$ centered at points of $\mathcal{S}$ :

$$
\mathcal{C}_{r}=\left\{B_{2 r}\left(z_{1}\right), B_{2 r}\left(z_{2}\right), \ldots, B_{2 r}\left(z_{N}\right)\right\}
$$

Consider a family of cylinders:

$$
\mathcal{Q}_{r}=\left\{Q_{r}\left(z_{1}\right), Q_{r}\left(z_{2}\right), \ldots, Q_{r}\left(z_{N}\right)\right\}
$$

For all sufficiently small $r$ we have $Q_{r}\left(z_{i}\right) \subset B_{2 r}\left(z_{i}\right)$.

\footnotetext{
${ }^{5}$ In fact, from the condition (1.4) and integrability of $|u|^{3}$ and $|p|^{3 / 2}$ it follows that the set $\mathcal{S}$ must be bounded. Therefore we have

$$
\mathcal{S} \subset\left(T_{1}, T_{2}\right) \times B(0, \tilde{R})
$$
}

for some $\tilde{R}>0$. 


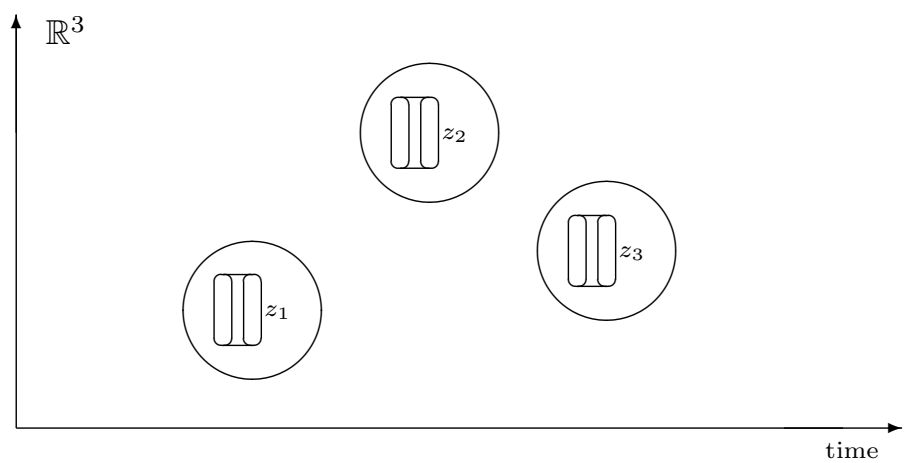

Since the parabolic cylinders are disjoint we obviously have

$$
\sum_{i=1}^{N(\mathcal{S}, r)} \int_{Q_{r}\left(z_{i}\right)}|u|^{4}+|p|^{2} \leq \int_{\Omega_{T}}|u|^{4}+|p|^{2}<\infty .
$$

Since points $z_{1}, z_{2}, \ldots, z_{N}$ are singular it follows from (1.4) that for all $i=1,2, \ldots, N$ we have

$$
\varepsilon \leq \frac{1}{r^{2}} \int_{Q_{r}\left(z_{i}\right)}|u|^{3}+|p|^{3 / 2} .
$$

From Hölder's inequality we get

$$
r^{3 / 4} \varepsilon \leq\left(\int_{Q_{r}\left(z_{i}\right)}|u|^{4}\right)^{3 / 4}+\left(\int_{Q_{r}\left(z_{i}\right)}|p|^{2}\right)^{3 / 4} .
$$

Combining (3.1) and (3.2) we obtain, for $\epsilon=(\varepsilon / 2)^{4 / 3}$,

$$
\operatorname{\epsilon r} N(\mathcal{S}, r)<\infty \text {. }
$$

If $d_{B}(\mathcal{S})$ was greater than 1 then for some $d>1$ we would have $N(\mathcal{S}, r) \geq r^{-d}$. Then the left hand side of (3.3) would tend to infinity, contradicting the integrability of $|u|^{4}+|p|^{2}$ on $\Omega_{T}$.

Acknowledgment. The author would like to thank Prof. James Robinson and Prof. John Gibbon for helpful comments.

\section{REFERENCES}

[1] L. Caffarelli, R.V. Kohn, and L. Nirenberg, Partial regularity of suitable weak solutions of the Navier-Stokes equations, Commun. Pure Appl. Math., 35, 771-831, 1982.

[2] K. Falconer, Fractal Geometry: Mathematical Foundations and Applications, 2nd edition, Chichester, John Wiley \& Sons, 2003.

[3] C.L. Fefferman, Existence and smoothness of the Navier-Stokes equation, 2000. http://www.claymath.org/millennium/Navier-Stokes Equations/navierstokes.pdf.

[4] G.P. Galdi, An introduction to the Navier-Stokes initial-boundary value problem, Fundamental directions in Mathematical Fluid Dynamics, Birkhauser, Basel, 1-70, 2000. 
[5] J.G. Heywood, Epochs of regularity for weak solutions of the Navier-Stokes equations in unbounded domains, Tohoku Math. J., 40, 293-313, 1988.

[6] E. Hopf, Über die Anfangswertaufgabe für die hydrodynamischen Grundgleichungen, Math. Nachr., 4, 213-231, 1951.

[7] A.A. Kiselev and O.A. Ladyzhenskaya, On existence and uniqueness of solutions of nonstationary problem of the flow of viscous, incompressible fluid, (in Russian), Izwiestia Akademii Nauk CCCP, 655-680, 1957.

[8] R.V. Kohn, Partial regularity and the Navier-Stokes equations, Lecture Notes in Num. Appl. Anal., 5, 101-118, Nonlinear PDE in Applied Science U.S. - Japan Seminar, Tokyo, 1982.

[9] A. Kufner, O. John, and S. Fucik, Function Spaces, Academia, Prague, 1977.

[10] I. Kukavica, The fractal dimenson of the singular set for solutions of the Navier-Stokes system, Nonline., 22, 2889-2900, 2009.

[11] O. Ladyzhenskaya, The Mathematical Theory of Viscous Incompressible Flows, 2nd Edition, Gordon and Breach, New York, 1968.

[12] O. Ladyzhenskaya and G. Seregin, On partial regularity of suitable weak solutions to the three dimensional Navier-Stokes equations, J. Math. Fluid Mech., 1, 356-387, 1999.

[13] J. Leray, Essai sur le mouvement dun fluide visqueux emplissant lespace, Acta Math., 63, 193-248, 1934.

[14] J.C. Robinson and W. Sadowski, Decay of weak solutions and the singular set of the threedimensional Navier-Stokes equations, Nonlin., 20, 1185-1191, 2007.

[15] J.C. Robinson and W. Sadowski, Almost-everywhere uniqueness of Lagrangian trajectories for suitable weak solutions of the three-dimensional Navier-Stokes equations, Nonlin., 22, 2093-2099, 2009.

[16] J.C. Robinson and W. Sadowski, On the dimension of the singular set of solutions to the Navier-Stokes equations, Commun. Math. Phys., 309(2), 497-506, 2012.

[17] J.C. Robinson, Dimensions, Embeddings, and Attractors, Cambridge Tracts in Mathematics, 2011.

[18] V. Scheffer, Turbulence and Hausdorff dimension, in Turbulence and Navier-Stokes equations, Springer LNM, 565, 174-183, 1976.

[19] J. Serrin, On the interior regulariy of weak solutions of the Navier-Stokes equations, Arch. Rat. Mech. Anal., 9, 187-191, 1962.

[20] Z. Skalak and P. Kučera, Regularity of pressure in the neighbourhood of regularity points of weak solutions of the Navier-Stokes equations, Appl. Math., 48(6), 573-586, 2003. 